\section{Highly expressed genes within hippocampal sector CA1: implications for the physiology of memory}

\author{
Michael A. Meyer \\ Department of Neurology, Sisters \\ Hospital, Buffalo, NY, USA
}

\begin{abstract}
As the CAl sector has been implicated to play a key role in memory formation, a dedicated search for highly expressed genes within this region was made from an on-line atlas of gene expression within the mouse brain (GENSAT). From a data base of 1013 genes, 16 were identified that had selective localization of gene expression within the CA1 region, and included Angpt2, ARHGEF6, CCK, Cntnap1, DRD3, EMP1, Epha2, Itm2b, Lrrtm2, Mdk, PNMT, Ppm1e, Ppp2r2d, RASGRP1, Slitrk5, and Sstr4. Of the 16 identified, the most selective and intense localization for both adult and post-natal day 7 was noted for ARHGEF6, which is known to be linked to non-syndromic mental retardation, and has also been localized to dendritic spines. Further research on the role played by ARHGEF6 in memory formation is strongly advocated
\end{abstract}

\section{Introduction}

Although the anatomy of new memory formation within the brain has been clarified, with the anatomic focus narrowed down to the CA1 region of the hippocampus, the physiologic and biochemical mechanisms that make this happen within the CAl region remain elusive. Confirming basic science studies in animals that CA1 is critical to new memory formation, a human autopsy study published in 1986 linked selective post-hypoxic damage to CA1 with clinically evident memory impairments; as noted by the authors, the affected patient showed marked anterograde amnesia, little if any retrograde amnesia, and showed no signs of cognitive impairment other than memory. ${ }^{1}$ Subsequent high resolution magnetic resonance imaging (MRI) studies in Alzheimer's disease confirm the impact that CAl atrophy has on memory performance; ${ }^{2}$ two other studies on transient global amnesia (TGA) patients with MR defined hippocampal ischemic events clearly show how focal damage to CA1 disrupts not only place memory but time dependent memory features including autobiographical memory. 3,4

One of the most elegant studies linking CA1 to memory is the optogenetic work of Deisseroth and colleagues, ${ }^{5}$ who were able to immediately and reversibly silence excitatory gluatamatergic CA1 electrical activity using a stereotactically implanted light sensitive lentiviral vector encoding $e N p H R 3.1$ fused inframe to enhanced yellow fluorescent protein (eNpHR3.1- EYFP) under control of the calcium/calmodulin-dependent protein kinase IIa (CaMKIIa) promoter which is selective and specific for glutamate neurons. The authors concluded that contextual fear memory recall, even weeks after training, can be reversibly abolished by temporally precise optogenetic inhibition of CAI and found that long-term memory retrieval normally depends on the hippocampus but can adaptively shift to alternate structures [...] we confirm the remotetimescale importance of the anterior cingulate cortex $(A C C)$ and implicate $C A 1$ in $A C C$ recruitment for remote recall.

Clearly, CA1 is the anatomic focus for memory investigations as neuronal activity from CA1 is critical in memory formation and retrieval. However, the biochemical events leading to the CA1 memory trace remains elusive and therefore became the physiologic focus of this investigation to identify those genes which are selectively and intensely expressed within the CA1 region. By searching a mouse brain histological expression atlas of 1013 genes, a set of 16 genes was found that met these criteria, with the most selectively intense expression found for the ARHGEF6 gene at both the post-natal day 7 and adult stage.

\section{Materials and Methods}

The Gene Expression Nervous System Atlas (GENSAT) project, was the main resource for this on-line atlas search. ${ }^{6}$ Details on the technical aspects of the GENSAT project for tissue processing of EGFP transgenic mice, as well as histochemical details are found at: http://www. gensat.org/HistologyProtocol.pdf.

As outlined in the histology core protocol, transgenic mice were kept until adulthood at 42 days, and then sacrificed for histologic processing, sectioning and EGFP immunolabelling with rabbit IgG anti-GFP followed by immunostaining with a secondary antibody (Biotin conjugated anti-rabbit IgG).

Using the original GENSAT on-line data base, all 1013 gene entries were inspected for histochemical evidence of selectively intense localization of activity to the CA1 region in sagittal sections from adult mice; diamino benzadine (DAB) was the chromogen and parasagittal section number 6 was used for uniform comparison of genes (Figure 1); similar sections of mouse brain at post-natal day 7 (P7) were also individually reviewed as well
Correspondence: Michael Andrew Meyer, Department of Neurology, Sisters Hospital, 2157 Main Street, Buffalo, NY 14214, USA

Tel.: + 1.716.862.2750.

E-mail: michaelandrewmeyer@gmail.com

Key words: memory, hippocampus, CA1, gene, atlas.

Conflict of interests: the author declares no potential conflict of interests.

Received for publication: 9 March 2014.

Accepted for publication: 7 April 2014.

This work is licensed under a Creative Commons Attribution NonCommercial 3.0 License (CC BYNC 3.0).

(C)Copyright M.A. Meyer, 2014

Licensee PAGEPress, Italy

Neurology International 2014; 6:5388

doi:10.4081/ni.2014.5388

with attention focused on finding consistently strong patterns of CA1 expression that were found not only in adult but at the P7 stage as well. The original parasagittal images of the 16 selected genes were enlarged to show just the CA1 region as shown in Figure 1, with Kyte \& Doolittle hydropathy plots made for the gene's amino acid sequence (http://web.expasy.org/ protscale/) and shown along the left and right hand outer panels of Figure 1.

\section{Results and Discussion}

High activity was selectively localized to CA1 for the following 16 genes: Angpt2, ARHGEF6, CCK, Cntnap1, DRD3, EMP1, Epha2, Itm2b, Lrrtm2, Mdk, PNMT, Ppm1e, Ppp2r2d, RASGRP1, Slitrk5, and Sstr4 (Figure 1).

Selectively intense localization CA1 was noted for Angpt2, which is otherwise known as angiopoietin 2. Staining was found within the neuropil as well as CA1 cell bodies, but was absent elsewhere in the brain except for the mild localization to the molecular layer of the cerebellum. Angpt2 is a protein that antagonizes the action of angiopoietin 1 (ANGPT1) and endothelial TEK tyrosine kinase (TIE-2, TEK) and interferes with the ability of ANGPT1 to remodel vessels partly due to its' ability to induce endothelial cell apoptosis. Not much is known about the action of Angpt2 within the brain; however, high-frequency stimulation of the ventrolateral thalamus over 14 days was found to modulate hippocampal gene expression, with Angpt2 being particularly affected.7

CCK displayed selectively intense CA1 stain- 
ing for the neuropil as well as the cell body layer with some staining noted for the frontal cortex and nearby entorhinal cortex. The octapeptide sulfated form of Cholecystokinin (CCK-8S) is the most common form of CCK within the brain and has been found to increase filopodia and spine density at in vitro day 7, 14 and 21 in hippocampal neurons of not only wild-type mice but also in double transgenic mice with mutations for the amyloid precursor protein and presenilin (APP/PS1); CI988, an antagonist of CCK-2 receptor, inhibits this effect of CCK-8S. 8 The memoryenhancing effects of CCK have been reviewed by Hadjiivanova et al. ${ }^{9}$

With regards to selectively intense CA1 localization of CNTNAP1 (contactin associated protein 1, also known as P190; CASPR; NRXN4; CNTNAP) it is important to note that Murai $e t$ $a l .{ }^{10}$ have reported a novel role for glycosylphosphatidyl inositol (GPI)-anchored contactin in hippocampal CA1 synaptic plasticity. These investigators found that contactin selectively supports paired-pulse facilitation (PPF) and NMDA (N-methyl-D-aspartate) receptordependent long-term depression (LTD) and that it is needed for proper targeting of the contactin-associated protein (Caspr/paranodin) towards the synapse. Additional findings were that contactin is needed for the proper distribution of the receptor protein tyrosine phosphatase beta (RPTPbeta)/phosphacan.

The investigators concluded that contactin plays a selective role in synaptic plasticity and identify PPF and LTD, but not LTP (long-term potentiation), as contactin-dependent processes.

As dopamine is a key neurotransmitter within the brain, it is very important to note that the D3 receptor subtype displayed selectively intense CA1 localization. The DRD3 dopamine receptor D3 is linked to $\mathrm{G}$ proteins that inhibit adenyl cycles, and displays genetic variations linked to essential tremor. Mice lacking dopamine D2 and D3 receptors have spatial working memory deficits: studies by Glickstein et al. ${ }^{11}$ have shown that mice lacking dopamine D2 and D3 receptors have spatial working memory deficits in addition to reduced c-fos responses to $D(1)$ agonist stimulation.

Another CA1 protein found in this search is the epithelial membrane protein 1 (EMP1), which is felt to be a novel tight junction protein of the blood-brain barrier. As noted by Bangsow et al., Epithelial Membrane Protein 1 expression was transiently induced in laser-capture microdissected rat brain vessels after a 20 -min global cerebral ischemia, in parallel with the loss of occludin immunoreactivity, suggesting a possible role in blood-brain barrier alterations in acute stroke. ${ }^{12}$ However, its potential role in hippocampus related memory formation remains elusive.

Ephrin receptor type A2 (EPHA2) was also identified in this search for CAl proteins. Ephrin and related receptor proteins are known to be linked to developmental events, especially for neuronal tissue, as well as to affect axon guidance, synaptic plasticity, and to promote long-term potentiation. As shown by Thundyil et al..$^{13}$ with a mouse middle cerebral artery occlusion model for stroke, neurological deficit scores and brain infarct volumes were significantly less in EphA2(-/-) mice compared with controls, leading the investigators to conclude that the data provide the first evidence that the EphA2 receptor directly contributes to blood-brain barrier damage and neuronal death following ischemic stroke. However, the role

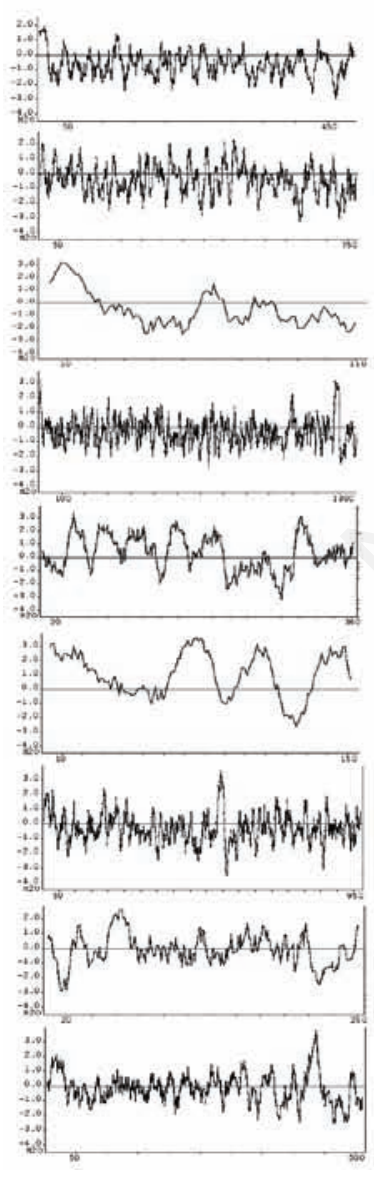

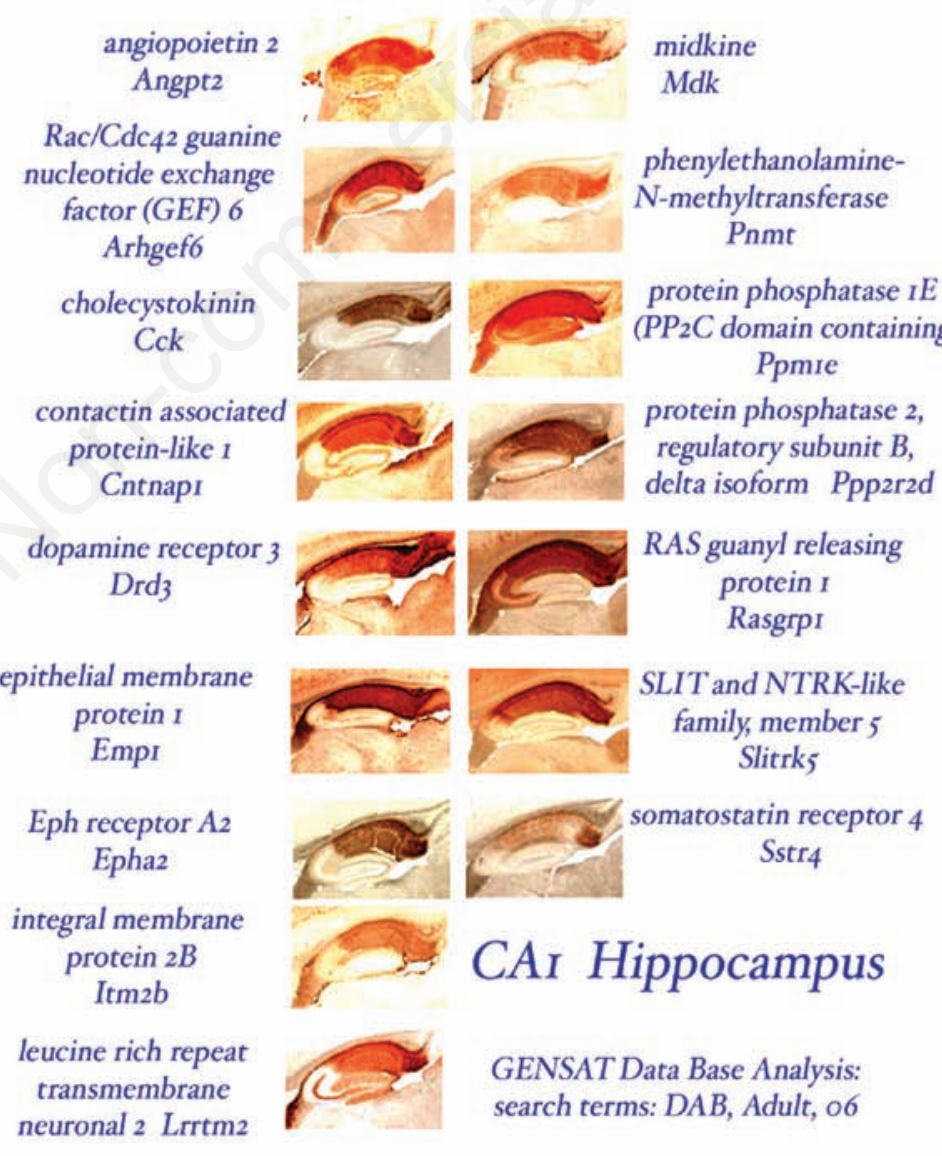

$\mathrm{Rac} / \mathrm{Cdc} 42$ guanine nucleotide exchange ctor (GEF)

Arhgef6 ystokinin protein-like $I$ Cntnap $\operatorname{Drd}_{3}$ protein I
EmpI

Eph receptor $A_{2}$ Epha2 gral membrane protein $2 B$ Itm2 $b$ transmembrane neuronal 2 Lrrtm2 ontactin associated midkine

phenylethanolamine$\mathrm{N}$-methyltransferase Pnmt

protein phosphatase $I E$ Ppmie

protein phosphatase 2 , regulatory subunit $B$, delta isoform Ppparzd

RAS guanyl releasing protein $r$ Rasgrpi

LIT and NTRK-like ily, member 5 Slitrk5 tin receptor 4 Sstr.

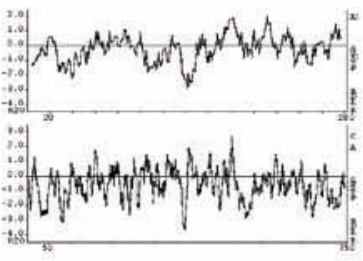

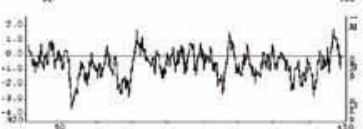

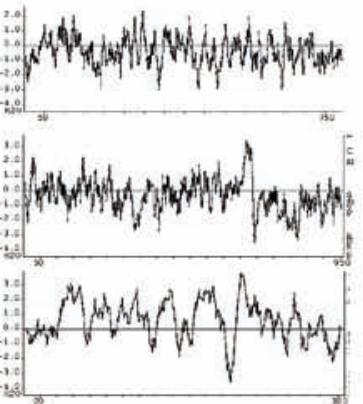

Figure 1. Sixteen highly expressed genes within adult mouse CA1 hippocampus are shown above, with hydropathy plots for the expressed protein shown as well. 
which Epha2 may play in hippocampus mediated memory formation remains unknown.

One of the most interesting proteins identified in this search was integral membrane protein 2B (ITM2B, also known as BRI; other aliases include FBD; ABRI; BRI2; E25B; E3-16; imBRI2; BRICD2B). ITM2B is a transmembrane protein which after $\mathrm{C}$-terminus processing by proteases, releases a small secreted peptide that blocks the deposition of beta-amyloid. Mutations which extend the length of the Cterminus end that then result in a larger size to the secreted peptide, have been found to cause two neurodegenerative diseases: familial British dementia (FBD) and familial Danish dementia (FDD). As reported by Tamayev et al. ${ }^{14}$ for their mouse model of FBD, the British mutation drastically reduces expression of mature BRI2 in both KI (knock-in) mice and human FBD brains. This deficit is associated with severe hippocampal memory deficits in $F B D(K I)$ mice. Remarkably, these animals showed no cerebral amyloidosis and tauopathy. Bri2(+/-) mice present memory deficits similar to those in $F B D(K I)$ animals. Collectively, these results indicate that the British BRI2 mutation underlies abnormal memory due to loss of BRI2 function and independently of histopathological alterations typically evident in advanced neurodegenerative disease.

Selectively intense staining of the CA1 neuropil was found for LRRTM2 (leucine rich repeat transmembrane neuronal 2) in addition to mild reactivity within the molecular layer of the dentate gyrus as well as the CA3 stratum lucidum. This protein is particularly relevant to the CA1 role in memory in that it is known to be a key regulator of excitatory synapse development and function. The studies of de Wit $e t$ al. have shown that LRRTM2 interacts with Neurexin1 and regulates excitatory synapse formation, 15 whereas Soler-Llavina et al. ${ }^{16}$ found that the protein was essential for maintenance of long-term potentiation of mature synapses on adult CA1 pyramidal neurons. Clinical correlates include a study by Kleffmann et al., ${ }^{17}$ who studied a patient with a small and restricted $5 q 31$ microdeletion and associated intellectual disability/developmental delay (ID/DD); they concluded [...] LRRTM2 as the most promising candidate gene for ID/DD due to its expression pattern, function as a key regulator of excitatory development, and interaction with Neurexin 1.

Midkine (MdK) is a growth factor involved in the development and repair of numerous types of tissues, especially neural tissue. Aside from a fairly prominent localization to the molecular layer of the cerebellum, this search identified selectively intense localization to the $\mathrm{CAl}$ region of the hippocampus. Although knockout mice without the $M d K$ gene encoding for the Midkine protein showed no gross abnormalities, the study of Nakamura et al. ${ }^{18}$ showed that they had working memory deficits that could be found at 4 weeks after birth, as revealed by Y-maze testing as well as the elevated plus-maze test; they concluded that MdK was an important regulator of hippocampal post-natal development.

Of particular interest was finding the epinephrine synthesis enzyme, PNMT, is selectively expressed within CA1 in addition to other patterns of intense expression within the dorsal medulla and mild activity within the thalamus. To investigate the selective impact of epinephrine on arousal and fear-related memory retrieval, Toth et al. studied PNMT knock-out mice unable to synthesize epinephrine and found that they had impaired conditioned fear response and startle reactivity. ${ }^{19}$

Although the $\mathrm{Ca}(2+) / c a l m o d u l i n-d e p e n d-$ ent protein kinase phosphatase-N (otherwise known as PPM1E or P0PX1) was found in this study to be selectively expressed within the CA1 region in an intense fashion, relatively little is known about this enzyme. PPM1E appears to be a protein phosphatase that specifically dephosphorylates and regulates multifunctional calcium and Calmodulin-regulated kinases but unique in that it localizes the nucleus (hence, -N). Ishida et al. ${ }^{20}$ found that by using gene knockdown studies in zebrafish, they were able to conclude that this enzyme was essential for the early development of the brain and spinal cord. As the pattern of expression for PPM1E was fairly intense and specific for CA1, further research on the functional role this enzyme may play in hippocampal physiology overall with specific inquiry to any possible role in memory formation.

On a possibly related note, another poorly studied protein phosphatase known as PPP2R2D (alias B-Delta, or B55-Delta) also showed strong and selective CA1 staining, yet relatively little Is known about it make functional correlations for the hippocampus. Of note, Hui-Feng Chen et al. ${ }^{21}$ estimated overall that Protein phosphatase 2A (PP2A) accounts for up to $1 \%$ of cellular protein, and that certain functional variants in haplotypes and nuclear factor 1 may regulate the transcription of the regulatory subunit gene $P P P 2 R 2 D$ (protein phosphatase 2 , regulatory subunit $\mathrm{B}$, delta).

Especially high levels of the Ras guanyl nucleotide-releasing protein (RasGRP) was found here in this study for the CA1 region. This protein is a Ras guanyl nucleotide exchange factor that is expressed prominently in the hippocampus, but also within the dendrites and cell bodies of fronto-parietal pyramidal neurons which can be traced to extend down into the internal capsule and beyond. Apparently, RasGRP can activate the Ras signal pathway in response to changes in diacylglycerol and possibly calcium; electron microscopy found RasGRP immunoreactivity in hippocampal pyramidal neuronal cell bodies and den- drites, where it appeared to be associated with microtubules. ${ }^{22}$

Another gene that seems to be strongly expressed within CA1 with prominent pyramidal cell staining as well as in the neuropil is the neuron-specific transmembrane protein, SLIT and NTRK-like protein-5 (Slitrk5). Not much is known about this gene with respect to brain physiology, but a gene knockout model shows that the deficiency leads to obsessivecompulsive like behaviors in mice, with excessive self-grooming observed along with hightened anxiety type behaviors, which seems to be relieved by the selective serotonin reuptake inhibitor fluoxetine; 23 the investigators feel that Slitrk5 acts as molecule at corticostriatal synapses. However, little is known overall about Slitrk5 and it's possible role in hippocampal physiology.

A particularly interesting finding in this study is detection of the Somatostatin receptor subtype 4 (SSTR4) as showing selectively intense expression within the CA1 region of the hippocampus. Studies by Gastambide et $a l .24$ found that this gene is involved in memory formation, as intra-hippocampal injections of the SSTR4 agonist (L-803,087) dramatically impaired place memory formation in a dosedependent manner yet agonists to Somatostatin receptor types 1,2 , or 3 had no effect. Other studies by Bito et al. ${ }^{25}$ concluded that [...] hippocampal functions of somatostatin might be mediated through diverse but selective second messenger systems activated via SSTR4 and reveal an unsuspected coupling of a neuronal SSTR subtype to a mitogenic signaling pathway.

The most interesting finding of all in this study is detection of ARHGEF6 as being selectively expressed within CAl in an intense manner for not only adult mice, but also for those examined at post-natal day 7 . This gene encodes the guanine nucleotide exchange factor PIX/Cool-2 for the Rho GTPases Racl and Cdc42, and activates Rho proteins by mediating the exchange of GDP for GTP; this is a critical function as Rho GTPases appears to act as important molecular switches by alternating between an inactive, GDP-bound state and the physiologically active form where GTP is bound. As mutations in five different $\mathrm{X}$-linked genes that encode proteins that interact with Rho GTPases (FGD1, OPHN1, PAK3, ARHGEF9 as well as ARHGEF6) are linked to intellectual disability, ${ }^{26}$ Ramakers et al.27 investigated ARHGEF6 knock-out mice and found that they exhibited impaired spatial and complex learning and had diminished behavioral control when subjected to mildly stressful situations. Furthermore, these ARHGEF6 knock-out mice showed an overall loss in spine synapses within the hippocampus; corresponding electrophysiological changes in CA1 neurons were also seen with reduced early-phase long-term 
potentiation and increased long-term depression. It therefore appears likely that ARHGEF6 may play a critical role in memory formation, and further research is clearly needed.

\section{Conclusions}

Although a prior study by Lein et al. ${ }^{28}$ attempted to identify genes selectively expressed within $\mathrm{CAl}$ and neighboring sectors of the hippocampus, it was limited by making the initial search through micro-array technology followed by in-situ hybridization (ISH) analysis of the more intensely expressed genes. This initial micro-array approach followed by ISH found genes displaying restricted expression within CA1 to include Nov and Bhlhb2 with genes displaying enriched expression in CA1 being Kl, Tmsb10, Etv1, Stac. Dusp1, AA591428, Egr1, and $P b x 3$. The current study of the GENSAT atlas under discussion here was conducted with remarkably different methods, and individually inspected the histological features of gene expression directly amongst 1013 genes as the initial discriminating factor rather than microarray signal analysis.

Hopefully, this study has provided a more comprehensive analysis to find those genes which have physiologic relevance to learning and memory; in this regard, a surprising number of genes were found out of the group of 16 identified that already have known links to memory, and includes ARHGEF6, SSRT4, PNMT, MdK, LRRTM2, ITM2B, DRD3, CNTNAP1 and CCK-8. Of particular interest is ARHGEF6, which has been tied to the important role that dendritic spines play in hippocampal mediated memory formation. ${ }^{26}$ As ARHGEF6 is an activator of Rho GTPases that regulate the actin cytoskeleton, it appears natural for it be able to influence dendritic spine morphogenesis; mutations within the ARHGEF6 gene also has been linked to mental retardation. ${ }^{27}$ Although ITM2B and LRRTM2 also appear to have important roles in memory, more research is clearly needed on the role which ARHGEF6 plays in hippocampal physiology.

\section{References}

1. Zola-Morgan S, Squire LR, Amaral DG. Human amnesia and the medial temporal region: enduring memory impairment following a bilateral lesion limited to field CA1 of the hippocampus. J Neurosci 1986;6:2950-67.

2. Kerchner GA, Deutsch GK, Zeineh M, et al. Hippocampal CA1 apical neuropil atrophy and memory performance in Alzheimer's disease. Neuroimage 2012;63:194-202.
3. Bartsch T, Schönfeld R, Müller FJ, et al. Focal lesions of human hippocampal CA1 neurons in transient global amnesia impair place memory. Science 2010;328:1412-5.

4. Bartsch T, Döhring J, Rohr A, et al. CA1 neurons in the human hippocampus are critical for autobiographical memory, mental time travel, and autonoetic consciousness. Proc Natl Acad Sci USA 2011;108: 17562-7.

5. Goshen I, Brodsky M, Prakash R, et al. Dynamics of retrieval strategies for remote memories. Cell 2011;147:678-89.

6. Gong S, Zheng C, Doughty ML, et al. A gene expression atlas of the central nervous system based on bacterial artificial chromosomes. Nature 2003;425:917-25.

7. Kádár E, Lim LW, Carreras G, et al. Highfrequency stimulation of the ventrolateral thalamus regulates gene expression in hippocampus, motor cortex and caudateputamen. Brain Res 2011;1391:1-13.

8. Zhang LL, Wei XF, Zhang YH, et al. CCK-8S increased the filopodia and spines density in cultured hippocampal neurons of APP/PS1 and wild-type mice. Neurosci Lett 2013;542:47-52.

9. Hadjiivanova C, Belcheva S, Belcheva I. Cholecystokinin and learning and memory processes. Acta Physiol Pharmacol Bulg 2003;27:83-8.

10. Murai KK, Misner D, Ranscht B. Contactin supports synaptic plasticity associated with hippocampal long-term depression but not potentiation. Curr Biol 2002; 12:181-90.

11. Glickstein SB, Hof PR, Schmauss C. Mice lacking dopamine D2 and D3 receptors have spatial working memory deficits. J Neurosci 2002;22:5619-29.

12. Bangsow T, Baumann E, Bangsow C, et al. The epithelial membrane protein 1 is a novel tight junction protein of the bloodbrain barrier. J Cereb Blood Flow Metab 2008;28:1249-60.

13. Thundyil J, Manzanero S, Pavlovski D, et al. Evidence that the EphA2 receptor exacerbates ischemic brain injury. PLoS One 2013;8:e53528.

14. Tamayev R, Giliberto L, Li W, et al. Memory deficits due to familial British dementia BRI2 mutation are caused by loss of BRI2 function rather than amyloidosis. $J$ Neurosci 2010;30:14915-24.

15. de Wit J, Sylwestrak E, O'Sullivan ML, et al. LRRTM2 interacts with Neurexin1 and regulates excitatory synapse formation. Neuron 2009;64:799-806.

16. Soler-Llavina GJ, Arstikaitis P, Morishita $\mathrm{W}$, et al. Leucine-rich repeat transmembrane proteins are essential for maintenance of long-term potentiation. Neuron 2013;79:439-46.

17. Kleffmann W, Zink AM, Lee JA, et al. 5q31 microdeletions: definition of a critical region and analysis of LRRTM2, a candidate gene for intellectual disability. Mol Syndromol 2012;3:68-75.

18. Nakamura E, Kadomatsu K, Yuasa S, et al. Disruption of the midkine gene (Mdk) resulted in altered expression of a calcium binding protein in the hippocampus of infant mice and their abnormal behaviour. Genes Cells 1998;3:811-22.

19. Toth M, Ziegler M, Sun P, et al. Impaired conditioned fear response and startle reactivity in epinephrine-deficient mice. Behav Pharmacol 2013;24:1-9.

20. Ishida A, Tsumura K, Oue M, et al. An active C-terminally truncated form of $\mathrm{Ca}$ $(2+) /$ calmodulin-dependent protein kinase phosphatase-N (CaMKP-N/PPM1E). Biomed Res Int 2013;2013:134813.

21. Chen HF, Lin LN, Chen YX, et al. Identification and functional analysis of variant haplotypes in the 5'-flanking region of protein phosphatase $2 \mathrm{~A}-\mathrm{B}$ gene. PLoS One 2012;7:e35524.

22. Pierret P, Vallée A, Mechawar N, et al. Cellular and subcellular localization of Ras guanyl nucleotide-releasing protein in the rat hippocampus. Neuroscience 2001;108: 381-90.

23. Shmelkov SV, Hormigo A, Jing D, et al. Slitrk5 deficiency impairs corticostriatal circuitry and leads to obsessive-compulsive-like behaviors in mice. Nat Med 2010;16:598-602.

24. Gastambide F, Viollet C, Lepousez G, et al. Hippocampal SSTR4 somatostatin receptors control the selection of memory strategies. Psychopharmacology (Berl) 2009;202:153-63.

25. Bito H, Mori M, Sakanaka C, et al. Functional coupling of SSTR4, a major hippocampal somatostatin receptor, to adenylate cyclase inhibition, arachidonate release and activation of the mitogen-activated protein kinase cascade. J Biol Chem 1994;269:12722-30.

26. Nodé-Langlois R, Muller D, Boda B. Sequential implication of the mental retardation proteins ARHGEF6 and PAK3 in spine morphogenesis. J Cell Sci 2006;119: 4986-93.

27. Ramakers GJ, Wolfer D, Rosenberger G, et al. Dysregulation of Rho GTPases in the Pix/Arhgef6 mouse model of X-linked intellectual disability is paralleled by impaired structural and synaptic plasticity and cognitive deficits. Hum Mol Genet 2012;21:268-86.

28. Lein ES, Zhao X, Gage FH. Defining a molecular atlas of the hippocampus using DNA microarrays and high-throughput in situ hybridization. J Neurosci 2004;24: 3879-89. 